\title{
Publisherl nfo
}

PublisherName : : BioMed Central

PublisherLocation : London

PublisherlmprintName : BioMed Central

\section{Predicting mortality in patients on continuous venovenous hemofiltration and hemodiafiltration}

\begin{tabular}{|lll|}
\hline & Articlel nfo \\
ArticlelD & $: 1598$ \\
ArticleDOI & $: 10.1186 /$ cc1550 \\
ArticleCitationID & $:$ E001 \\
ArticleSequenceNumber & $: 23$ \\
ArticleCategory & $:$ Non-peer-reviewed research \\
ArticleFirstPage & $: 1$ \\
ArticleLastPage & $: 8$ \\
ArticleHistory & $:$ RegistrationDate $:$ 2002-2-21 \\
& & OnlineDate \\
ArticleCopyright & $:$ BioMed Central Ltd2002 \\
ArticleGrants & $:$ & \\
ArticleContext & $:$ 130546611 \\
\hline
\end{tabular}


J eannette M Capella,Aff1

Corresponding Affiliation: Aff1

Email: jcapella@creighton.edu

Kevin R Keating, Aff2

Aff1 Attending, Department of Surgery, Creighton University, Omaha, USA

Aff2 Director, Surgical Critical Care, Hartford Hospital, Hartford, USA

\section{Abstract}

\section{Introduction}

The purpose of this study is to correlate levels of mortality in patients on CWH/D with the degree of illness as determined by the APACHE II score. We also identified variables that were part of the APACHE II score that had the most significant impact on outcome. No study has looked at this particular question. This could give physicians, patients and families better information on which to base care decisions.

\section{Aims}

To identify and quantify variables that predict mortality in critically ill patients on CWH/D.

\section{Methods}

\section{General Description}

The study was a retrospective descriptive analysis of all patients in the ICU's at Hartford Hospital who were on CWH/D between January 1997 and December 1999.

\section{Patients}

There were 83 patients on CWH/D during the specified time period with complete information. Those who were placed on this form of renal replacement therapy were considered too hemodynamically unstable to tolerate hemodialysis.

\section{Data}

Age, sex, past medical history, admission diagnosis, operations, number of failing organ systems and survival were recorded for all patients. The following data was obtained from the date of initiation of $\mathrm{CWH}$ /D: sodium, potassium, creatinine, $\mathrm{pH}, \mathrm{PaO} 2$, hematocrit, white cell count, Glasgow Coma Scale, temperature, heart rate, respiratory rate, and mean arterial pressure. The APACHE II score was calculated using the method described in Knaus et al. [5]

\section{Statistical Analysis}

Survivors and nonsurvivors were compared. Parametric data was analyzed using the student's t test with the Levene's test for equality of variance. The Mann-Whitney test was used for nonparametric data. A logistic regression analysis was done for the APACHE II score and age, after these were determined to be the most significant variables for determining mortality.

Results 
Of the eighty-three patients for whom we were able to obtain all necessary data, twenty-two (26.5\%) survived until hospital discharge. Table 1 describes the diagnoses in our population.

Table 1 Diagnoses in Survivors and Nonsurvivors on CWH/D

\begin{tabular}{|l|l|l|}
\hline Diagnosis & Survivors & Nonsurvivors \\
\hline Cardiac & 10 & 31 \\
\hline Gastrointestinal & 1 & 8 \\
\hline Sepsis/SI RS & 2 & 7 \\
\hline Pneumonia & 2 & 4 \\
\hline Vascular & 3 & 2 \\
\hline Liver & 0 & 4 \\
\hline Trauma & 2 & 2 \\
\hline Pancreas & 0 & 2 \\
\hline Hematologic & 1 & 1 \\
\hline Neurologic & 1 & 0 \\
\hline Total & 22 & 61 \\
\hline
\end{tabular}

Fifty-nine $(71.1 \%)$ were surgical patients and twenty-four $(28.9 \%)$ were medical. Survival was $28.8 \%$ in surgical patients and $20.1 \%$ in medical patients, which was not statistically significant $(p=0.221)$.

Twenty-two (26.5\%) patients had acute on chronic renal failure and sixty-one $(73.5 \%)$ had acute renal failure. Survival was $31.8 \%$ in the former and $24.6 \%$ in the latter; again, this was not significant ( $p=$ 0.281). There were fifty-three $(63.4 \%)$ males and thirty $(36.6 \%)$ females; there was no difference in the male: female ratio between survivors and nonsurvivors.

All of the patients had at least three-organ system failure, including cardiovascular, renal and respiratory failure. Eleven patients had four organ system failure (nine had hepatic failure and two had anoxic brain injury) and all but one of these died.

At the time the decision was made to initiate $\mathrm{CWH} / \mathrm{D}$, the APACHE II score and the age were significantly different $(p<0.01)$ between survivors and nonsurvivors (Table 2$)$. The Chronic Health Problem score, $\mathrm{pH}$, and creatinine were also significantly different $(\mathrm{p}<0.05)$.

Table 2 Comparison of Parameters for Survivors and Nonsurvivors

\begin{tabular}{|l|l|l|l|l|c|}
\hline & \multicolumn{2}{|c|}{ Survivors } & \multicolumn{2}{c|}{ Nonsurvivors } & $p$ \\
\hline Parameter & Mean & SD & Mean & SD & \\
\hline APACHE II score & 24.6 & 4.0 & 28.2 & 5.7 & $\mathbf{0 . 0 0 8}$ \\
\hline Age & 54.6 & 16.7 & 64.7 & 14.9 & $\mathbf{0 . 0 1 0}$ \\
\hline Chronic Health & 2.1 & 2.3 & 3.4 & 2.2 & $\mathbf{0 . 0 2 3}$ \\
\hline Problem score & & & & & \\
\hline Temperature (C) & 36.7 & 1.2 & 37.0 & 0.8 & 0.311 \\
\hline Heart Rate & 94 & 20 & 96 & 20 & 0.735 \\
\hline Mean Arterial Pressure & 75 & 19 & 71 & 16 & 0.620 \\
\hline & & & & &
\end{tabular}




\begin{tabular}{||l|l|l|l|l|c|}
\hline Respiratory Rate & 14 & 5 & 16 & 7 & 0.550 \\
\hline PH & 7.38 & 0.06 & 7.34 & 0.08 & $\mathbf{0 . 0 2 6}$ \\
\hline Sodium & 136 & 8 & 138 & 6 & 0.353 \\
\hline Potassium & 4.4 & 0.7 & 4.5 & 0.8 & 0.574 \\
\hline Creatinine & 4.9 & 1.4 & 3.9 & 1.7 & $\mathbf{0 . 0 1 6}$ \\
\hline White Cell Count & 15.4 & 9.7 & 15.5 & 12.1 & 0.968 \\
\hline Hematocrit & 28.9 & 2.6 & 29.5 & 3.6 & 0.424 \\
\hline Glasgow Coma Scale & 8 & 3 & 8 & 3 & 0.663 \\
\hline Number Organs Failed & 3.0 & 0.2 & 3.2 & 0.4 & 0.413 \\
\hline
\end{tabular}

Boldface indicates significance with a p value of 0.050 .

Logistic regression analysis of the APACHE II score and age showed that APACHE II score less than or equal to 25 versus greater than 25 , and age less than 60 versus greater than or equal to 60 were the most useful cutoff values. The odds of dying was 4.8 times higher for a person requiring CWH/D if they were 60 years old or more than if they were younger than 60 . The odds of dying was 3.7 times higher if their APACHE II score was greater than 25. Furthermore, an APACHE II score greater than 25 and age greater than or equal to 60 years (thirty-two patients) predicted mortality in $91 \%$ of these patients.

\section{Discussion}

Mortality in patients requiring CWH/D in the ICU is quite high because, by definition, these patients have at least two-organ system failure. In our series, all had at least three-system failure. However, most physicians and patients would probably agree that even a mortality of $60-80 \%$ does not constitute futile care. We set out to identify variables which might help us define subgroups in which CWH/D is futile.

The APACHE II score was chosen because it is a broadly accepted and commonly used score for determining the degree of illness of patients in the ICU. Ideally, this score would have been calculated during the first twenty-four hours of admission to the ICU. However, the vast majority of our patients did not have complete data at that point in time. We were, however, able to obtain complete data sets for all of our patients at the time CWH/D was started.

Also, the APACHE II score is ideally used to describe large groups of patients. The relatively small size of our group, therefore, weakens our conclusions. Nevertheless, this is the largest analysis of this type ever completed. Previous studies have looked at very small numbers of patients with specific diagnoses.

Gender and a history of chronic renal failure did not have an impact on mortality. Neither was there a difference between surgical and medical patients. As expected, nonsurvivors had a significantly higher APACHE II score at the time of initiation of CWH/D than survivors. Age was the most important component of the APACHE II score; survivors were significantly younger than nonsurvivors. Survivors tended to have a higher $\mathrm{pH}$ and a lower Chronic Health Problem score than the nonsurvivors. And interestingly, the creatinine tended to be higher in the survivors; we cannot explain this finding. A larger sample size (two hundred) would be needed to include $\mathrm{pH}$, the Chronic Health Problem score and creatinine in a logistic regression analysis.

A much larger multiinstitutional analysis or a metaanalysis would be required to more accurately define how acidosis, creatinine level, previous chronic health problems and specific diagnoses affect the ultmate outcome. However, this paper does show that the APACHE II score at the time of the initiation of CWH/D and the age of the patient were important predictors of mortality in patients requiring CWH/D. Whether further therapy, including $\mathrm{CWH} / \mathrm{D}$, constitutes futile care in these patients, will still need to be decided by patients, their families and their physicians. We hope that this data will be of use in making this decision. 


\section{Introduction}

Patients who are ill enough to require admission to the ICU and who require CWH/D have a mortality rate of $60-80 \%[1,2,3,4]$. The few studies, which have looked at outcomes in patients on CWH/D, have focused on particular subgroups of patients, and included burn victims, liver transplants and heart transplants $[2,3,4]$. They did not make any attempt to objectively determine how ill the patients were to begin with. Swartz et al. compared patients needing CWH/D versus hemodialysis (HD)[1]. They believed that patients needing HD were not as ill as those on CWH/D and thought the difference in mortality could be accounted for by this fact. However, they were unable to determine the (Acute Physiology and Chronic Health Evaluation) APACHE II scores and objectively confirm this suspicion.

The purpose of this study is to correlate levels of mortality in patients on CWH and CWHD with the degree of illness as determined by the APACHE II score. We also identified variables that were part of the APACHE II score that had the most significant impact on outcome. No study has studied this particular question. This could give physicians, patients and families better information on which to base care decisions, and would result in better utilization of resources and cost containment.

\section{Materials and Methods}

\section{General Description}

The study was a retrospective descriptive analysis of all patients in the medical and surgical ICU's at Hartford Hospital who were on CWH/D between January 1997 and December 1999. Approval of the study was obtained from the Institutional Review Board at Hartford Hospital; informed consent was waived.

\section{Patients}

There were 85 total patients on $\mathrm{CWH} / \mathrm{D}$ during the specified time period. The charts of two of the patients were unavailable; these were excluded. Those who were placed on this form of renal replacement therapy were considered too hemodynamically unstable to tolerate hemodialysis.

\section{Data}

Age, sex, past medical history, admission diagnosis, operations, number of failing organ systems and survival were recorded for all patients. The following data was obtained from the date of initiation of CWH/D: sodium, potassium, creatinine, pH, PaO2, hematocrit, white cell count, Glasgow Coma Scale, temperature, heart rate, respiratory rate, and mean arterial pressure. The APACHE II score was calculated using the method described in Knaus et al. [5] An attempt was made to obtain the same data from admission to the ICU as well, but very few patients had all of the necessary data. 


\section{Statistical Analysis}

Survivors and nonsurvivors were compared. Parametric data was analyzed using the student's $t$ test with the Levene's test for equality of variance. The Mann-Whitney test was used for nonparametric data, including the APACHE II score and its component Chronic Health Problem score. A logistic regression analysis was done for the APACHE II score and age, after these were determined to be the most significant variables for determining mortality.

\section{Results}

Of the eighty-three patients for whom we were able to obtain all necessary data, twenty-two (26.5\%) survived until hospital discharge. Forty-one (49.3\%) were cardiac patients (Table 1 ) and included those with coronary artery disease, myocardial infarction, congestive heart failure and valvular disease. Of the patients with SIRS, one had sepsis from necrotizing fasciitis. The gastrointestinal diagnoses included obstruction and bleeding. All of the liver patients were in failure and awaiting transplant. One pancreas patient had pancreatitis and one had a carcinoma. All of the vascular patients had an aortic aneurysm or occlusive disease. One trauma patient had rhabdomyolysis from pressure necrosis. The others had severe multisystem injuries after motor vehicle accidents.

Fifty-nine $(71.1 \%)$ were surgical patients and twenty-four $(28.9 \%)$ were medical. Survival was $28.8 \%$ in surgical patients and $20.1 \%$ in medical patients, which was not statistically significant $(p=0.221)$. Twentytwo $(26.5 \%)$ patients had acute on chronic renal failure and sixty-one $(73.5 \%)$ had acute renal failure. Survival was $31.8 \%$ in the former and $24.6 \%$ in the latter; again, this was not significant $(p=0.281)$. There were fifty-three $(63.4 \%)$ males and thirty $(36.6 \%)$ females; there was no difference in the male:female ratio between survivors and nonsurvivors.

All of the patients had at least three-organ system failure, including cardiovascular, renal and respiratory failure. In this study we defined patients with cardiovascular failure as those who needed inotropic, pressor and/or mechanical support. Those with renal failure required renal replacement therapy. Those with hepatic failure were coagulopathic, encephalopathic and/or jaundiced. Those with respiratory failure required ventilatory support. Those with brain failure included those with anoxic brain injury. Eleven patients had four organ system failure (nine had hepatic failure and two had anoxic brain injury) and all but one of these died. This last was a man who had anoxic brain injury after a cardiac arrest who eventually went to a nursing home. The difference in the number of organ system failures in the survivors and nonsurvivors was not significant $(p=0.413)$.

At the time the decision was made to initiate $C W H / D$, the APACHE II score and the age were significantly different $(p<0.01)$ between survivors and nonsurvivors (Table 2$)$. The Chronic Health Problem score, $\mathrm{pH}$, and creatinine were also significantly different $(\mathrm{p}<0.05)$.

Logistic regression analysis of the APACHE II score and age showed that APACHE II score less than or equal to 25 versus greater than 25, and age less than 60 versus greater than or equal to 60 were the most useful cutoff values. The odds of dying was 4.8 times higher for a person requiring CWH/D if they were 60 years old or more than if they were younger than 60 . The odds of dying was 3.7 times higher if their APACHE II score was greater than 25. Furthermore, an APACHE II score greater than 25 and age greater than or equal to 60 years (thirty-two patients) predicted mortality in $91 \%$ of these patients. 


\section{Discussion}

Mortality in patients requiring $\mathrm{CWH} / \mathrm{D}$ in the ICU is quite high. This is not to imply that $\mathrm{CWH} / \mathrm{D}$ is the cause. The high mortality is expected, since these patients, by definition, have at least two-organ system failure. In our series, all had at least three-system failure. However, most physicians and patients would probably agree that even a mortality of $60-80 \%$ does not constitute futile care. We set out to identify variables, which might help us define subgroups in which $\mathrm{CWH} / \mathrm{D}$ is futile.

The APACHE II score was chosen because it is a broadly accepted and commonly used score for determining the degree of illness of patients in the ICU. Ideally, this score would have been calculated during the first twenty-four hours of admission to the ICU. However, the vast majority of our patients did not have complete data at that point in time. We were, however, able to obtain complete data sets for all of our patients at the time $\mathrm{CWH} / \mathrm{D}$ was started.

Also, the APACHE II score is ideally used to describe large groups of patients. The relatively small size of our group, therefore, weakens our conclusions. Nevertheless, this is the largest analysis of this type ever completed. Previous studies have looked at very small numbers of patients with specific diagnoses. Oeseph et al. studied heart transplant patients; those needing CWH or HD (six on CWH and two on HD) had a mortality of $63.5 \%$. These were too few to compare survivors and nonsurvivors. Mortality in the patients without renal failure was 9\%. Leblanc et al. studied sixteen burn patients with renal failure; all received $\mathrm{CWH} / \mathrm{D}$. Mortality was $82 \%$; the mean APACHE II score at admission was 18.4 and the mean burn surface area was 58\%. Again, there were not enough patients to compare survivors and nonsurvivors. Fiore et al. looked at eleven liver transplant patients requiring CWH. A copy of the manuscript was not available for evaluation. There were not enough patients in our population to determine if specific diagnoses had an impact on survival.

Gender and a history of chronic renal failure did not have an impact on mortality. Neither was there a difference between surgical and medical patients. As expected, nonsurvivors had a significantly higher APACHE II score at the time of initiation of CWH/D than survivors. Age was the most important component of the APACHE II score; survivors were significantly younger than nonsurvivors. Survivors tended to have a higher $\mathrm{pH}$ and a lower Chronic Health Problem score than the nonsurvivors. And interestingly, the creatinine tended to be higher in the survivors; we cannot explain this finding. A larger sample size would be needed to include $\mathrm{pH}$, the Chronic Health Problem score and creatinine in a logistic regression analysis. A power analysis determined that two hundred patients would be required and we have not had that many on CWH/D at Hartford Hospital.

A much larger multiinstitutional analysis or a metaanalysis of data from several institutions would be required to more accurately define how acidosis, creatinine level, and previous chronic health problems affect the ultmate outcome. Also, such a study would enable us to determine if specific diagnoses have any impact on survival.

However, this paper does show that the APACHE II score at the time of the initiation of CWH/D and the age of the patient were important predictors of mortality in patients requiring CWH/D. An APACHE II score greater than 25 and age greater than or equal to 60 years (thirty-two patients) predicted mortality in 91\% of these patients. Whether further therapy, including CWH/D, constitutes futile care in these patients, will still need to be decided by patients, their families and their physicians. We hope that this data will be of use in making this decision. 


\section{References}

1. Swartz RD, Messana JM, Orzol S, Port FK: Comparing continuous hemofiltration with hemodialysis in patients with severe acute renal failure. Am J Kidney Dis. 1999, 34: 424-432.

2. Ouseph R, Brier ME, Jacobs AA, Erbeck KM: Continuous venovenous hemofiltration and hemodialysis after orthotopic heart transplantation. Am J Kidney Dis. 1998, 32: 290-294.

3. Leblanc $M$, Thibeault $Y$, Querin S: Continuous haemofiltration and haemodiafiltration for acute renal failure in severely burned patients. Burns. 1997, 23: 160-165. 10.1016/S0305-4179(96)00085-X.

4. Fiore G, Donadio PP, Gianferrari P, Santacroce C, Guermani A: CWH in postoperative care of liver transplantation. Minerva Anestesiol. 1998, 64: 83-87.

5. Knaus WA, Draper EA, Wagner DP, Zimmerman JE: APACHE II: a severity of disease classification system. Crit Care Med. 1985, 13: 818-829. 